\title{
New High-Resolution Low-Voltage and High Performance Analytical FIB/SEM System
}

\author{
Jaroslav Jiruše, Miloslav Havelka, Martin Haničinec, Jan Polster, Tomáš Hrnčír
}

TESCAN Brno, s.r.o., Libušina třída 1, Brno, Czech Republic

We have developed a new analytical tool GAIA3, combining ultra-high resolution SEM with high resolution FIB column. Electron column configuration comes from well-established TESCAN's three lens optical system with Schottky field-emission gun. The new single-pole immersion objective lens [1, 2] creates strong magnetic field in the chamber and dramatically decreases optical aberrations compared to the conventional lens, thereby it improves the SEM resolution down to $1 \mathrm{~nm}$ at $15 \mathrm{kV}$ and $1.4 \mathrm{~nm}$ at 1 $\mathrm{kV}$, see Figure 1. Combination of the intermediate lens (IML) and objective lens allows to work in various display modes (e.g. for the best resolution, the largest field of view or highest depth of focus), including a field-free mode for magnetic samples observation. GAIA3 instrument also benefits from the TESCAN's beam deceleration technology which allows automatic control of the electron landing energy down to $50 \mathrm{eV}$ (manually down to $0 \mathrm{eV}$ ) by applying negative voltage on the sample and further improves the resolution at low voltages. The new module provides a sample discharge-and-touch alarm protection.

High performance FIB column with gallium ion source enables to reach high resolution of $2.5 \mathrm{~nm}$ at 30 $\mathrm{kV}$ [3]. A new non-magnetic design protects from influence on the magnetic field of the immersion SEM objective lens. A mono-isotopic ion source avoids beam splitting in the presence of strong magnetic field in the chamber. Charge accumulation on the surface of non-conductive samples caused by incident ions can be neutralized using integrated electron flood gun or using electron beam of SEM.

Sophisticated detection system enables to continuously control FIB milling process and to image the material and topographic contrast of fabricated structures. Besides traditional chamber detectors for detection of secondary (SE), backscattered (BE) and transmitted (TE) electrons and secondary ions (SI), the InBeam SE and InBeam BE detectors placed in the column give free space over the sample for unlimited FIB work and various analytical techniques.

Comfortable control of the whole system is ensured by user-friendly software, which includes integrated In-Flight Beam Tracing ${ }^{\mathrm{TM}}$ technology. It is used not only for SEM control, e.g. very accurate computation of working distance, magnification and all optical parameters together with continuous control of the beam spot size and beam current of the SEM, but it newly enables to compute and optimize the ion beam too. New software module AutoSlicer is an easy-to-use tool for automated FIB cross sectioning and TEM lamella preparation, see Figure 2. AutoSlicer uses an innovated fast cross section milling approach increasing the milling speed more than 2.5 times. It is a part of TESCAN DrawBeam software that allows drawing of patterns in CAD-like GUI for electron and ion beam lithography. The patterns are generated by ultra-fast scanning with pixel dwell time down to $20 \mathrm{~ns}$ [4]. 


\section{References:}

[1] Z. Shao, PSD Lin, Rev. Sci. Instrum. 60 (1989), p. 3434.

[2] J. Jiruse et al., Microsc. Microanal. 19 (Suppl 2), 2013, p. 1302.

[3] A. Delobbe, O. Salord and P. Sudraud, EFUG meeting, 2011.

[4] The research leading to these results has received funding from the European Union Seventh

Framework Program [FP7/2007-2013] under grant agreement $n^{\circ} 280566$, project UnivSEM.

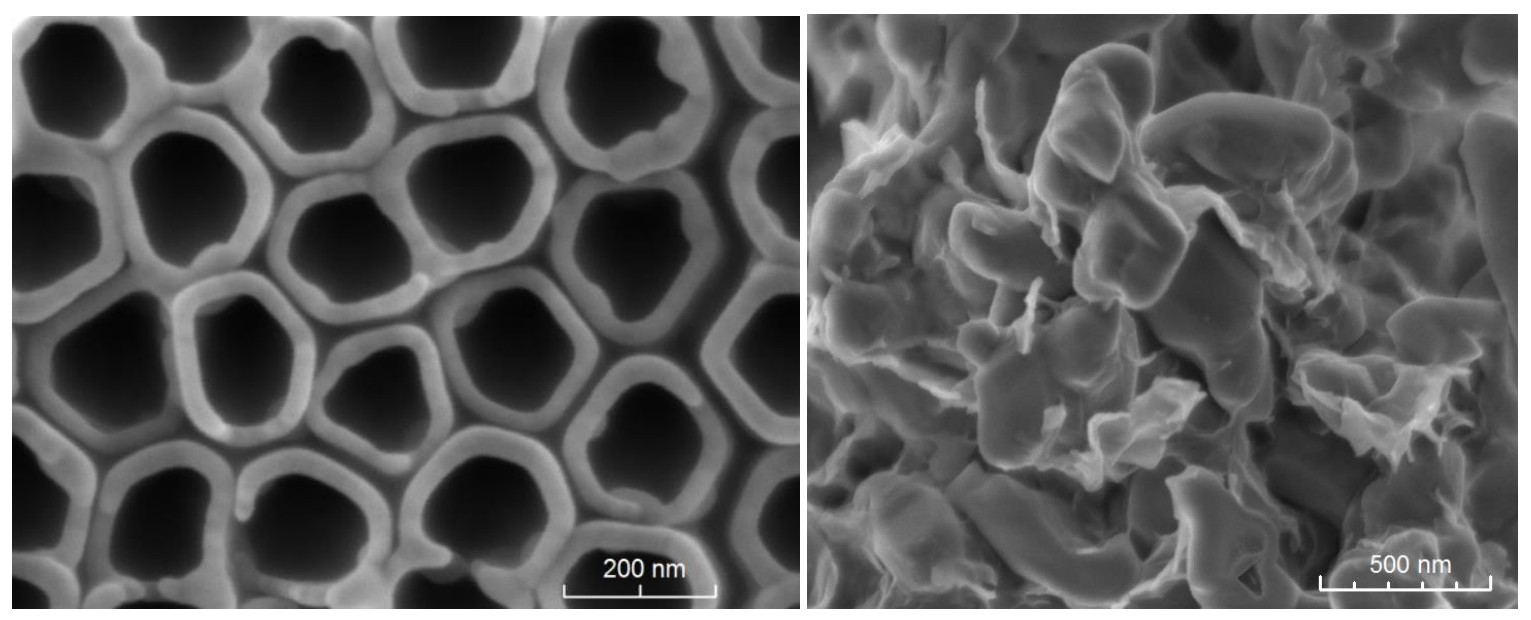

Figure 1. Ultra-high resolution at low voltages: $\mathrm{TiO}_{2}$ nanotubes at $1 \mathrm{kV}$ (left) and graphen with $\mathrm{LiFePO}_{4}$ at $2 \mathrm{kV}$ (right).
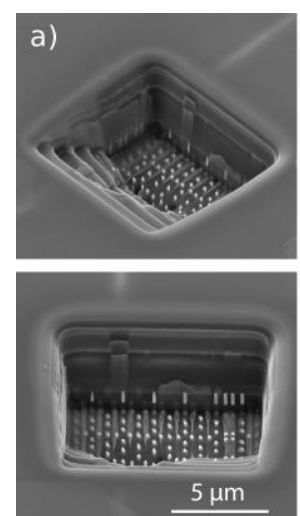


Figure 2. Comparison of different approaches for cross section preparation (left): (a) basic top-down "staircase" strategy, (b) a single-pass cross section preparation with high re-deposition, (c) a fully optimized cross section object with more than 2.5 times higher milling speed, the highest depth and the best shape without re-deposition artifacts. All the three objects were milled using $12 \mathrm{nA}$ FIB current at $30 \mathrm{keV}$. Automated TEM lamella preparation with innovative fast cross section milling approach (right). 